\title{
Fuzzy System Based Routing in computer Networks
}

\author{
Hisham Shaalan Saiwan Alshaheen \\ Thi-Qar University- College of Science \\ Email: hish772000@yahoo.com
}

\begin{abstract}
$\underline{\text { Abstract }}$
This paper presented fuzzy system to solve the routing problem. The proposed method is used two different metrics; link delay and congestion level to make routing decision and to manage congestion control. The fuzzy system is decides the selection level of neighbor node. So, the selection level of each neighbor node can be determined. The routing system selects best neighbor node, that has less value of link delay and congestion level from source to till destination. In this paper a performance improvement of fuzzy system is presented by adding a congestion level to together with link delay. These two input will be considered in route selection decision using fuzzy logic.
\end{abstract}

Keywords: Fuzzy system; Routing algorithm; and Mamadani Type.

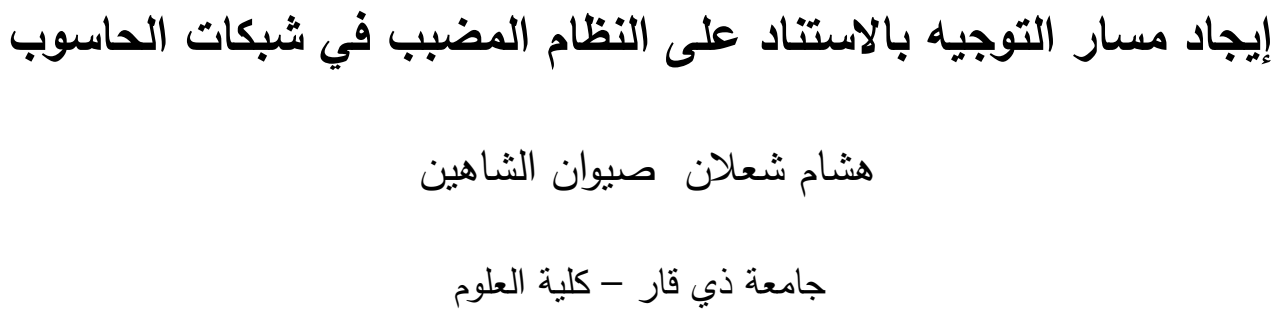

في هذا البحث تم اعتماد النظام المضبب في حل مشكلة التوجيه . حيث تم استخدام معيارين مختلفين هما التأخير الرابط ومستوى الاكتظاظ لصنع القرار ومعالجة مشكلة الاكتظاظ ـ حيث نم انشاء برنامج لكي يقرر مستوى الاختيار للعقدة المجاورة التي تحقق الثرطين


على التأخيرالرابط ومستوى الاكتظاظ باستخدام النظام المضبب. 


\section{1- Introduction}

Knowledge based systems such as fuzzy systems have been proven efficiency in various applications especially when dealing with impression and non statistical uncertainty such as decision making support and intelligent control, the fuzzy system is often typified by an inference system that executes the fuzzy reasoning rules in the system, input membership functions that are used for fuzzification of the input variables, and defuzzification to get the crisp outputs [1,2].

Fuzzy logic can be used efficiently as a solution technique for routing problem in computer networks, to improve the performance [3]. Routing is one of the major components of network control techniques that determine the overall network performance in terms of quality and quantity of the delivered service [4]. Fuzzy logic has been applied to routing in communication networks. The fuzzy system can be integrated into a complete routing system to define fuzzy cost to send a packet on each link of the path to the destination node based on different metrics $[3,5,6]$. This paper proposed a fuzzy system to manage congestion control with solving routing problem.

\section{Routing Algorithm}

Routing algorithms are methods for finding the best way from a node source to another node destination [3]. The routing algorithm represents the heart of the routing protocol, it is a method or a formula that is stored in the router's memory to compute the path that would best serve to transport the data from the source to the destination, acting as the traffic officer of the router by making decisions for the router concerning the best paths for data $[1,7]$.

\section{Congestion}

Congestion is defined as a state in which network performance is degraded in some way because of an excessive number of packets in transit within the network, a greater delay encountered by packets in traversing the network.
Congestion may local, confined to limited region of a network, or more seriously, it may be general throughput a network[3,8]. It is important of routing algorithms not only to get high aggregated bandwidth of flow assignment, but also to minimize network congestion to avoid excessive delay. The problem of optimal path from source to destination is minimizing the increase in the total network congestion, that may be affected on links of the selected route. The goal of any routing and congestion control mechanism is to avoid such congested network areas[3].

\section{Fuzzy system}

A fuzzy system is a system that uses a collection of fuzzy membership functions and rules [3]. A fuzzy system basically consists of four components: a knowledge base, an inference engine (or decision making unit), fuzzifier and defuzzifier units[8]. Figure(1) shows the diagram of a fuzzy system.



Figure (1) the diagram of a fuzzy system

\subsection{Fuzzy Inference}

The input-output mapping of the fuzzy control model is performed through the fuzzy inference system assigned with a rule base. The main components of a fuzzy inference engine are [10];

1. Input variables with its membership functions

2. Fuzzy rule base with linguistic rule curves

3. Output variables with its membership functions 
There are three main steps involved in FIS;

1. Fuzzification

2. Inferencing

3. Defuzzification

Fuzzification involves assignment of the membership function to the input value and calculating the degree of membership of that element to the fuzzy set. As already mentioned, the two most popular methods of inferencing are the Mamdani and Sugeno type of inference systems [10].In this study a Mamdani type is used.

In Mamdani type each rule takes the form [1]:

is $\mathrm{B}^{\mathrm{k}}$

$$
\mathrm{R}_{\mathrm{k}} \text { : If } \mathrm{x}_{1} \text { is } \mathrm{A}_{1}{ }^{\mathrm{k}} \text { and .... and } \mathrm{x}_{\mathrm{n}} \text { is } \mathrm{A}_{\mathrm{n}}{ }^{\mathrm{k}} \text { then } \mathrm{y}
$$

where $\mathrm{A}_{\mathrm{i}}^{\mathrm{K}}, \mathrm{B}^{\mathrm{K}}$ is the antecedent linguistic term associated to each input variable $\mathrm{x}_{\mathrm{i}}$ from $\mathrm{n}$ input variables, output variable $y$. Usually the output is computed by the max-min inference, which establishes that the activation degree of each rule is computed by applying the min operator to the antecedents, and the operator max to aggregate the outputs to get the final output value[2].

\subsection{Fuzzy Rule Base}

This is the heart of fuzzy inference engine. This performs the actual mapping or inferencing between the input and output fuzzy sets. The fuzzy rule base is a collection of rule curves represented in the form of linguistic variables. A rule is represented in the form of an If-Then statement. The rule contains the fuzzy input set in the antecedent and fuzzy output set in the consequent. A general rule is in the form of

\section{If $A$ is $x$ Then $B$ is $y$}

In the above rule, ' $x$ ' is a fuzzy set of the input variable $A$ and ' $y$ ' is the fuzzy set of the output variable B. Aggregation operators such as AND/OR are used when there exists more than one input or output variable.[10]

\subsection{Defuzzification}

The output set after undergoing implication and composition is in the form of a fuzzy set. It has to undergo the defuzzification process to output a crisp output value. There are many methods for defuzzification few of which are Center of Area method, Mean of Maxima method, Largest of Maximum method etc. The defuzzification method used in this study is the centre of area method or popularly known as the centroid method. The final crisp output value is calculated by determining the center of area of the output fuzzy set[10].

Crisp value $=\sum_{i=1}^{n} c_{i} A_{i} / \sum_{i=1}^{n} A_{i}$

Where $\mathrm{n}$ is the number of activation rules, $\mathrm{c}_{\mathrm{i}}$ is the center of membership function, $A_{i}$ is area of activation part of membership function[3].

\section{The Proposed Method of Routing Using Fuzzy Logic}

The proposed approach based on fuzzy system is used to solve the routing problem. The routing decision based on the produced routes fuzzy from two fuzzy input parameters:

\section{1- Link delay(sec)):}

The link delay refers to the length of time required to move a packet from source to neighbor nodes. The link delay metric used for selecting route, since less value of link delay represent a best link.

\section{2- Level of congestion:}

Level of congestion is the number of packets traveling on the link in the computer network. The level of congestion is determined through knowledge received from other nodes. Level of congestion is also information that is reported by other nodes, with values based on the average amount of traffic traveling through the computer network.

In the proposed method, the fuzzy system is located at each node of the computer network in Figure(2) to make a local routing decision. In the proposed fuzzy system has two input variables, they are link delay and congestion level of neighbor node and one output variable is the neighbor selection level. In this way both minimum link delay and minimum congestion level, providing an optimized network response. 
The computer network which is considered in this work is illustrated in Figure(2). The linguistic values with triangular membership functions of these variables are:
- (Short, Medium, Long) of congestion level.

- (Good, Medium, Bad) of neighbor selection level .

- (Low, Medium, High) of link delay.

While the fuzzy rule base is illustrated in Table 1.

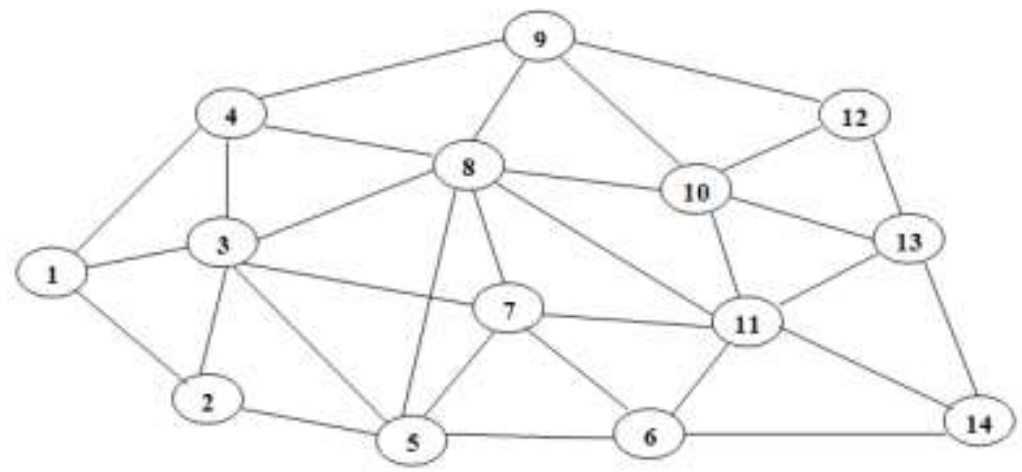

Figure(2) Computer network of 14 nodes

Table(1) the rules base of the fuzzy system

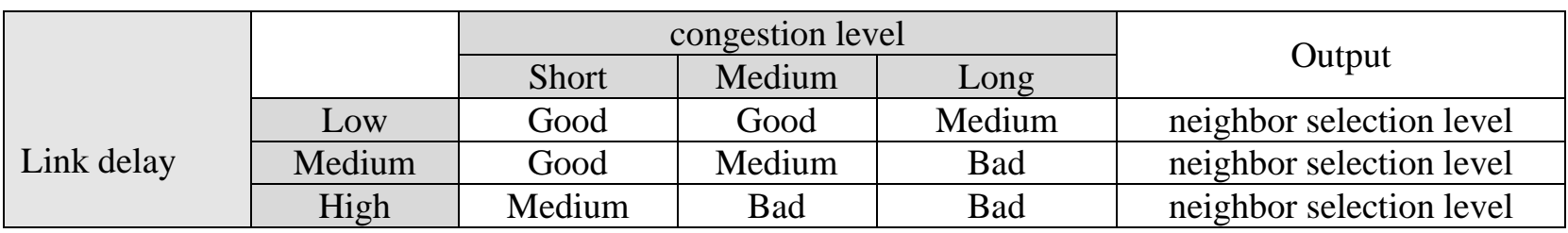

\section{Simulation Results}

To evaluated the efficiency of the proposed method that used in this study, it has been applied to the example of computer network in the Figure(2) The implementation has realized in Matlab. The proposed fuzzy system have two linguistic input variable, one linguistic output and number of rules( nine rules). The rules base of the fuzzy system is Mamdani type. The center of area method is used at the defuzzification of the system. The value of membership function of inputs/output which are show in Figure(3) and relation between inputs and output is show in Figure(4). For example, in computer network, if node 1 has packet to send to node 13, the fuzzy system at node 1 selects best neighbor node that has less value link delay with less value of the congestion level, that one of neighbor nodes $(2,3,4)$ to node 1 .The fuzzy system decides selection level of this neighbor node. So, the selection level of each neighbor node can be determined. Some of the results of the fuzzy system for computer network in Figure (2) is listed in Tables(2). 


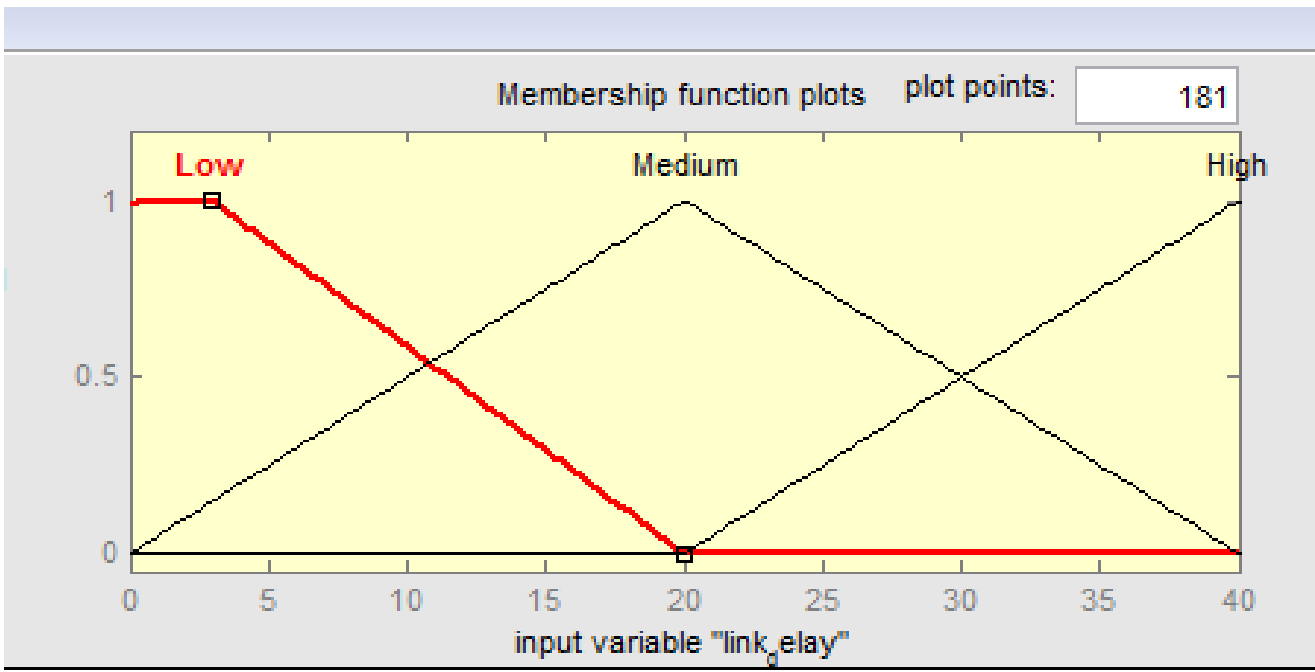

(a): Link delay

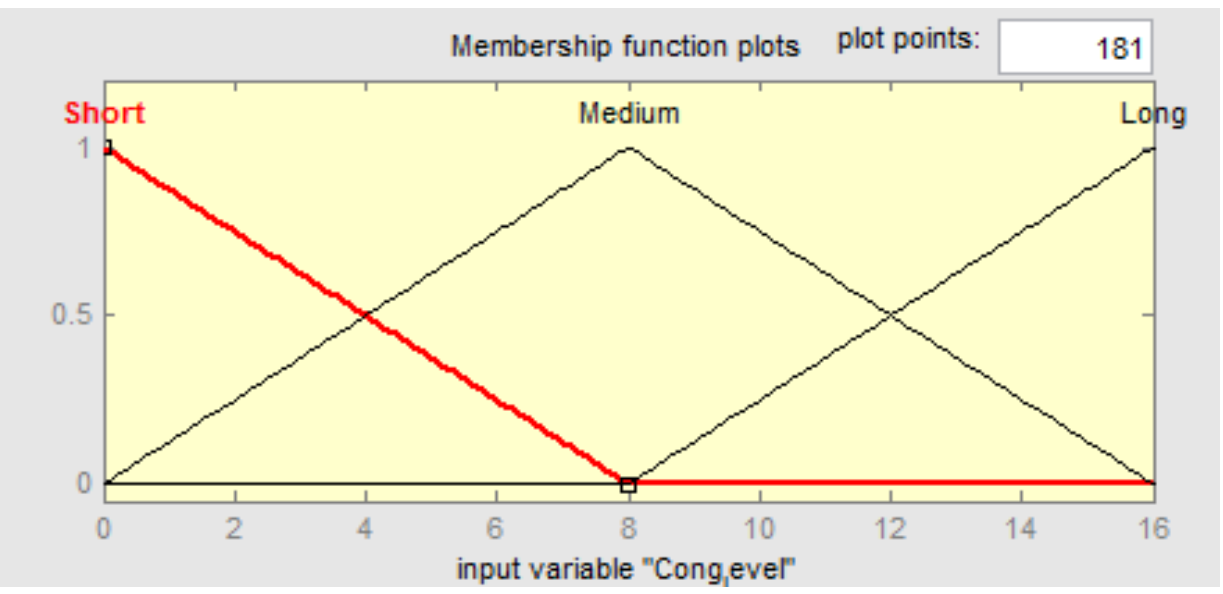

(b): congestion level

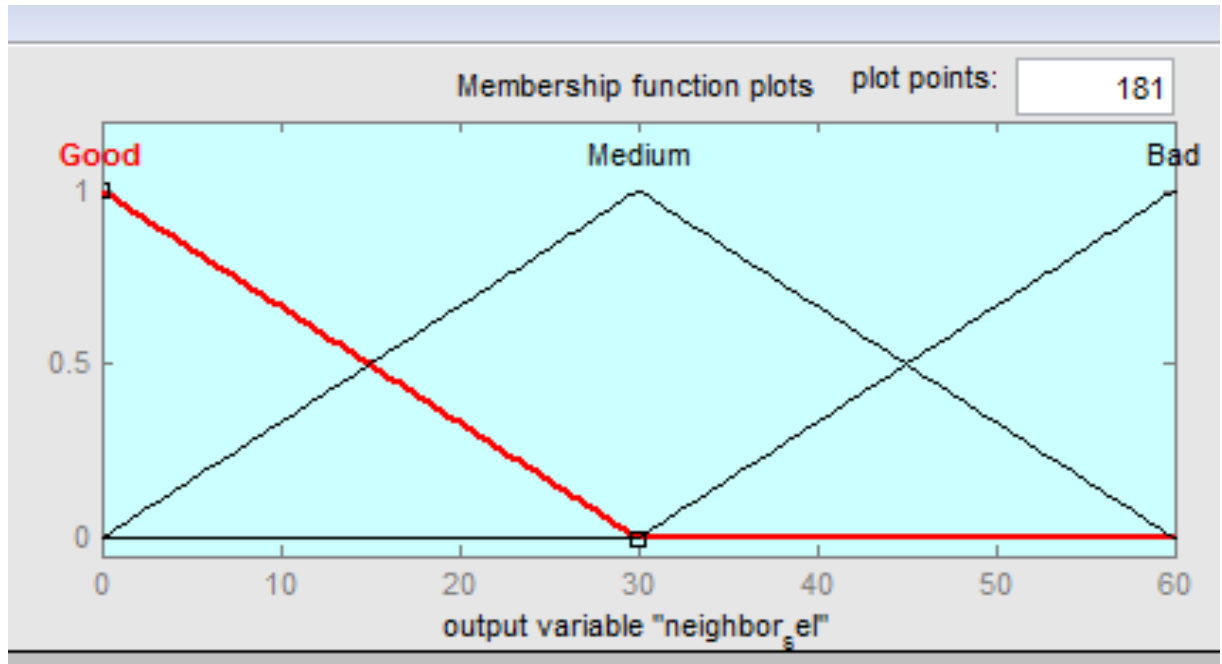

(c): neighbor selection level

Figure (3) Membership function of fuzzy system: (a): Link delay.(b):congestion level, (c):neighbor selection level. 


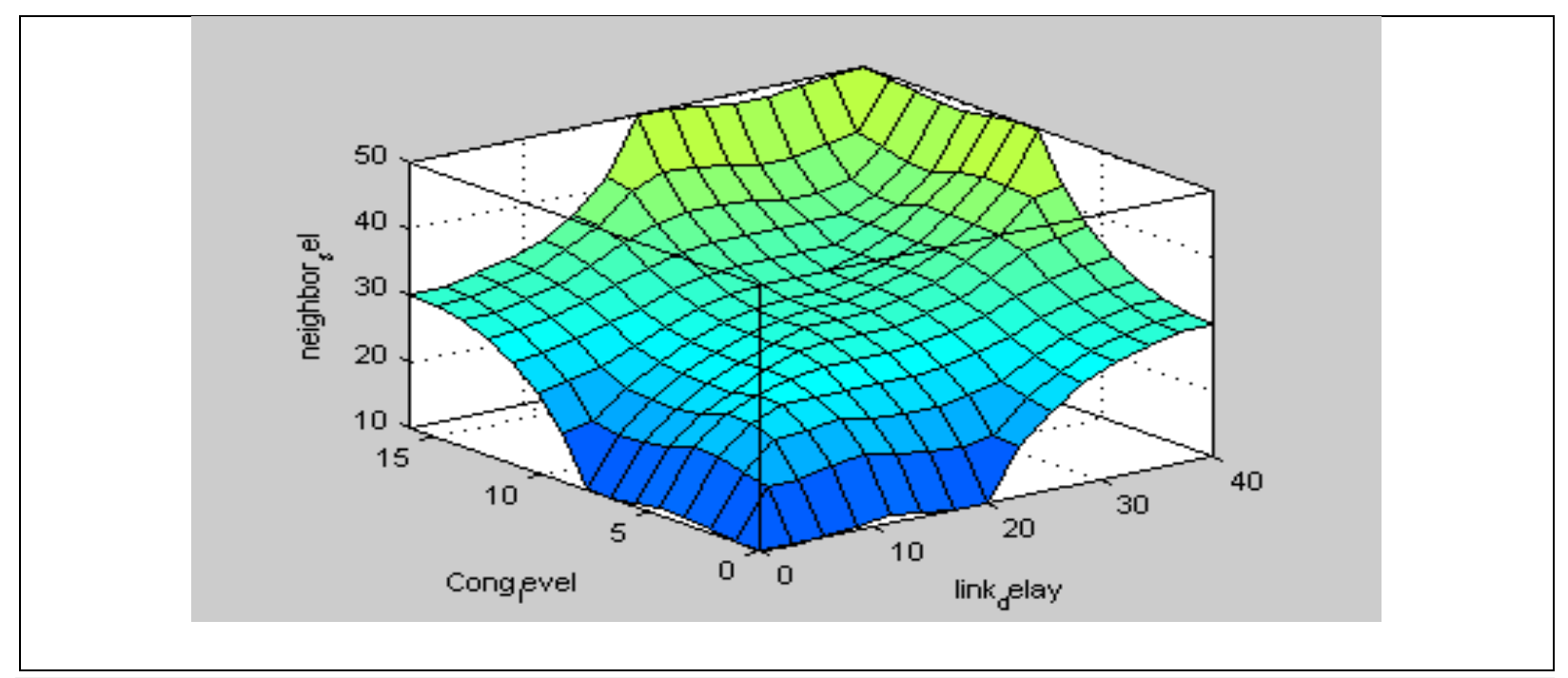

Figure (4) relation of fuzzy system

Table (2) some of the result of the fuzzy system for the computer network

\begin{tabular}{|c|c|c|}
\hline \multicolumn{2}{|c|}{ Input variables } & Output variable \\
\hline Link delay & Congestion level & Neighbor selection level \\
\hline 2.4 & 0.5 & 13.5991 \\
\hline 4 & 0 & 9.842 \\
\hline 12.4 & 5 & 27.2012 \\
\hline 3.6 & 8.6 & 18.5854 \\
\hline 0.25 & 7.2 & 10.7492 \\
\hline 16.2 & 4.7 & 27.4681 \\
\hline 25 & 10 & 30.9638 \\
\hline 32 & 13.5 & 36.6079 \\
\hline 37 & 16 & 49.9815 \\
\hline
\end{tabular}

\section{Conclusion}

The proposed fuzzy system consider two input variable the link delay and congestion level to make routing decision. The results is obtained in this paper is good results (neighbor selection level) because we used two metrics (link delay and Congestion level)this way help us to avoid a congested network areas. When information increases about the computer networks such as through put (data rate) of links, and the numbers of hops adding with above metric, a best decision we can obtain. 


\section{$\underline{\text { References }}$}

[1]- Taqaw Odey Fahad, "Adaptive routing in MANETs using fuzzy system", Master Thesis, Basrah University, 2011.

[2]- C. C. Lee, "Fuzzy Logic In Control Systems: Fuzzy Logic Controller- Part I", IEEE Transactions on Systems, Man, and Cybernetics, Vol. 20, No. 2, 1990, pp. 404418.

[3]- K. A. Naser, "Computer Network Routing Using Neural Networks And Fuzzy Systems", Ph.D. thesis, University of Basrah, Department of Computer science, 2007.

[4]- A. Pasupuleti, A.V. Mathew, N. Shenoy and S. A. Dianat," A Fuzzy System for Adaptive Network Routing " Rochester Institute of Technology,Rochester, NY 14623, USA,2004.

[5]- E. Aboelela and C.Douligeris, "Routing in Multimetric Networks Using A Fuzzy Link Cost", The second IEEE Symposium on computers and Communications, 1997.

[6]- M. Kara, H. Karabelli and N.Duru, "Fuzzy Based Routing in Packet Switching Networks", International XII. Turkish Symposium on Artificial Intelligence and Neural Networks, Tainn, 2003.

[7]- D. Medhi and K. Ramasamy, " Network Routing: Algorithms, Protocols, And Architectures", Morgan Kaufmann Publishers, 2007.

[8]-D. Davies, D. Barber, W. Price and C. Solomonides, "Computer Networks and Their Protocols ", J. Wiley and Sons Ltd, New York, 1979.

[9]- T. J. Ross, "Fuzzy Logic With Engineering Applications", Second Edition, Wiley, 2004.
[10]- B. Vallamsundar," Congestion Control for Adaptive Satellite Communication Systems with Intelligent Systems", Master thesis in Systems Design Engineering, University of Waterloo, Canada, 2007. 